\title{
СРАВНИТЕЛЬНЫЙ АНАЛИЗ БИОТОКСИЧНОСТИ ЭССЕНЦИАЛЬНЫХ ЭЛЕМЕНТОВ В ОТНОШЕНИИ ПРЕДСТАВИТЕЛЕЙ МИКРОБИОМА КИШЕЧНИКА
}

\author{
О.А. Филиппова *, Ю.А. Плотникова, Л.Р. Мусина, Ж.Б. Сулименова, Сизенцов Я.А. \\ Оренбургский государственный университет, г. Оренбург, пр. Победы, 13 \\ *e-mail: olga.filippova98@mail.ru
}

РЕЗЮМЕ. Сбалансированное соотношение эссенциальных элементов является ключевым показателем функционирования организма на физиологически значимом уровне. В системе биодоступности элементов в организм важную роль выполняет микробиом кишечника, активно участвующий в конструктивных метаболических процессах. Полученные в процессе экспериментов данные свидетельствуют о наличии выраженной биотоксичности соединений $\mathrm{Fe}, \mathrm{Cu}$ и $\mathrm{Zn}$ в отношении всех исследуемых штаммов и относительной толерантности в отношении высоких концентраций $\mathrm{Mg}$ и $\mathrm{Mn}$. Распределение показателей резистентности неоднородно и имеет индивидуальные характеристики устойчивости в отношении исследуемых элементов.

КЛЮЧЕВЫЕ СЛОВА: микробиом, эссенциальные элементы, биотоксичность.

\section{COMPARATIVE ANALYSIS OF BIOTOXICITY OF ESSENTIAL ELEMENTS IN RELATION TO REPRESENTATIVES OF THE INTESTINAL MICROBIOME}

\author{
O.A. Filippova*, Ya.A. Plotnikova, L.R. Musina, Zh.B. Sulimenova, Yu.A. Sizentsov \\ Orenburg State University, 13, Pobedy ave, 460018, Orenburg, Russia \\ *e-mail: olga.filippova98@mail.ru
}

\begin{abstract}
A balanced ratio of essential elements is a key indicator of the functioning of the body at a physiologically significant level. In the system of bioavailability of elements in the body, an important role is played by the intestinal microbiome, which is actively involved in constructive metabolic processes. The data obtained during the experiments indicate the presence of pronounced biotoxicity of the $\mathrm{Fe}, \mathrm{Cu}$ and $\mathrm{Zn}$ compounds in relation to all studied strains and relative tolerance in relation to high concentrations of $\mathrm{Mg}$ and $\mathrm{Mn}$. The distribution of resistance indicators is heterogeneous and has individual characteristics of resistance in relation to the studied elements.
\end{abstract}

KEYWORDS: microbiome, essential elements, biotoxicity.

\section{ВВЕДЕНИЕ}

Ключевым показателем функционирования организма на физиологически значимом уровне является сбалансированное соотношение эссенциальных элементов. В системе биодоступности элементов в организм важную роль выполняет микробиом кишечника, активно участвующий в конструктивных метаболических процессах.

Цель работы - сравнительный анализ биотоксичности эссенциальных элементов в отношении представителей микробиома кишечника

\section{МАТЕРИАЛЫ И МЕТОДЫ}

Объектами исследования служили факультативно-анаэробной нормофлоры кишечника крыс (Enterococcus faecium, Lactobacillus acidophilus, Escherichia coli). В качестве регулирующих рост факторов использовали $\mathrm{FeSO}_{4}, \mathrm{MgSO}_{4}, \mathrm{MnSO}_{4}, \mathrm{CuSO}_{4}, \mathrm{ZnSO}_{4}$ общим критерием для выбранных соединений считали их высокий уровень диссоциации в водных растворах, позволяющий в короткие промежутки времени создать высокие концентрации активных форм катионов в субстрате. Оценку уровня толерантности исследуемых микроорганизмов проводили с использованием комбинации методик диффузии в агар и серийных разведений (Sizentsov et al., 2018). 
Таблица. Влияние биотоксичности исследуемых эссенциальных элементов на изоляты микробиома кищечника лабораторных животных, зона подавления роста в мм

\begin{tabular}{|c|c|c|c|c|c|}
\hline \multirow{2}{*}{$\begin{array}{c}\text { Соли } \\
\text { металлов }\end{array}$} & \multicolumn{2}{|c|}{ Концентрация } & \multicolumn{3}{|c|}{ Исследуемые штаммы } \\
\hline & $\mathrm{M}$ & мГ/мл & E. faecium & L. acidophilus & E. coli \\
\hline \multirow{4}{*}{$\mathrm{FeSO}_{4}$} & 2 & 750 & $22,500 \pm 0,444$ & $12,300 \pm 0,745$ & $27,300 \pm 0,577$ \\
\hline & 1 & 375 & $19,500 \pm 0,408$ & $9,800 \pm 0,484$ & $25,100 \pm 0,309$ \\
\hline & 0,5 & 187,5 & $14,800 \pm 0,111$ & $5,600 \pm 0,643$ & $23,800 \pm 0,200$ \\
\hline & 0,25 & 93,8 & $11,100 \pm 0,260$ & - & $20,200 \pm 0,146$ \\
\hline \multirow{4}{*}{$\mathrm{MgSO}_{4}$} & 2 & 660 & $21,700 \pm 0,741$ & - & $9,300 \pm 1,795$ \\
\hline & 1 & 330 & $10,100 \pm 2,568$ & - & - \\
\hline & 0,5 & 165 & $7,400 \pm 1,871$ & - & - \\
\hline & 0,25 & 82,5 & - & - & - \\
\hline \multirow{4}{*}{$\mathrm{MnSO}_{4}$} & 2 & 302 & $24,200 \pm 0,426$ & $18,000 \pm 0,258$ & $25,400 \pm 0,392$ \\
\hline & 1 & 151 & $18,000 \pm 0,337$ & $15,600 \pm 0,305$ & $18,800 \pm 0,435$ \\
\hline & 0,5 & 75,5 & - & $12,700 \pm 0,422$ & $10,800 \pm 0,377$ \\
\hline & 0,25 & 37,8 & - & - & - \\
\hline \multirow{4}{*}{$\mathrm{CuSO}_{4}$} & 2 & 318,4 & $32,300 \pm 1,336$ & $34,700 \pm 2,332$ & $32,300 \pm 3,281$ \\
\hline & 1 & 159,6 & $30,700 \pm 1,450$ & $31,000 \pm 0,580$ & $30,000 \pm 3,050$ \\
\hline & 0,5 & 79,8 & $25,700 \pm 0,330$ & $26,300 \pm 0,333$ & $26,300 \pm 0,333$ \\
\hline & 0,25 & 39,9 & $16,700 \pm 3,181$ & $19,300 \pm 1,200$ & $22,000 \pm 3,510$ \\
\hline \multirow{4}{*}{$\mathrm{ZnSO}_{4}$} & 2 & 323 & $33,300 \pm 1,665$ & $34,300 \pm 2,333$ & $31,300 \pm 1,681$ \\
\hline & 1 & 161,5 & $28,000 \pm 1,121$ & $32,300 \pm 1,330$ & $27,700 \pm 2,945$ \\
\hline & 0,5 & 80,8 & $25,700 \pm 1,872$ & $28,000 \pm 1,322$ & $26,300 \pm 2,210$ \\
\hline & 0,25 & 40,4 & $21,000 \pm 2,545$ & $24,000 \pm 1,421$ & $23,300 \pm 0,842$ \\
\hline
\end{tabular}

\section{РЕЗУЛЬТАТЫ И ОБСУЖДЕНИЕ}

Полученные в результате экспериментальных исследований данные свидетельствуют о выраженном ингибирующем эффекте высоких концентраций $\mathrm{Fe}, \mathrm{Cu}$ и $\mathrm{Zn}$ в отношении тест-организмов (таблица). Следует отметить видовую резистентность L. acidophilus в отношении высоких катионных нагрузок железа на субстрат в концентрации $0,25 \mathrm{M}$ и выраженной устойчивости к воздействию $\mathrm{Mg}(2 \mathrm{M})$.

Минимальные уровни токсичности исследуемых элементов зарегистрированы при взаимодействии изолятов с катионами $\mathrm{Mg}$ (E. coli в концентрации $1 \mathrm{M})$ и $\mathrm{Mn}$ (E. faecium в концентрации 0,5 M). Практическая значимость полученных результатов позволит использовать накопленные данные при создании селективных питательных сред для выделения и идентификации представителей микробиома, а также интерпретировать данные экспериментов in vivo, направленных на оценку влияния микробиома на элементный статус.

\section{ВЫВОДЫ}

Анализ данных позволяет предположить наличие стратегии детоксикации, направленной на ферментативную детоксикацию (аккумуляцию) металла до нетоксичной биологически активной формы (депонирование), на поверхностных компонентах клетки (Пищик и др., 2016).

\section{Список литературы / References}

1. Sizentsov A.N., Cherkasov S.V., Karpova G.V., Bibartseva E.V., Kvan O.V., Kunavina E.A., Levenets T.V., Strekalovskaya A.D. The technology of chemical compound biotoxicity assessment by the method of agar basins. International Journal of Mechanical Engineering and Technology (IJMET). 2018; 9(11): 455-46.

2. Пищик В.Н., Воробьев Н.И., Проворов Н.А., Хомяков Ю.В. Механизмы адаптации растений и микроорганизмов в растительно-микробных системах к тяжелым металлам. Микробиология. 2016; 85(3): 231-247 [Pishhik V.N., Vorob'ev N.I., Provorov N.A., Homjakov Ju.V. Mehanizmy adaptacii rastenij i mikroorganizmov v rasti-tel'no-mikrobnyh sistemah k tjazhelym metallam. Mikrobiologija. 2016; 85(3): 231-247]. 